\title{
Subliminal words activate semantic categories (not automated motor responses)
}

\author{
RICHARD L. ABRAMS \\ University of Washington, Seattle, Washington \\ MARK R. KLINGER \\ University of Alabama, Tuscaloosa, Alabama \\ and \\ ANTHONY G. GREENWALD \\ University of Washington, Seattle, Washington
}

\begin{abstract}
Semantic priming by visually masked, unidentifiable ("subliminal") words occurs robustly when the words appearing as masked primes have been classified earlierin practice as visible targets. It has been argued (Damian, 2001) that practice enables robust subliminal priming by automatizing learned associations between words and the specific motor responses used to classify them. Two experiments demonstrate that, instead, the associations formed in practice that underlie subliminal priming are between words and semantic categories. Visible words classified as pleasant or unpleasant in practice with one set of response key assignments functioned later as subliminal primes with appropriate valence, even when associations of keys with valences were reversed before the test. This result shows that subliminal priming involves unconscious categorization of the prime, rather than just the automatic activation of a practiced stimulus-response mapping.
\end{abstract}

It is well established that recent experience with a word facilitates its processing on the next occurrence. This repetition priming effect has lately become germane to the interpretation of unconscious, or subliminal, semantic priming, in which classification of a visible target word is affected by the category (congruent or incongruent) of an immediately preceding, visually masked, unidentifiable ("subliminal") prime word. Evidence accumulated over the last several years indicates that the effectiveness of subliminal words as primes is highly dependent on the recent practice of classifying those words in visible form. From this evidence, four conclusions are warranted.

First, in studies in which participants had previously classified subliminal primes in visible form (practiced words, hereafter), priming effects were robust and easily replicable (e.g., Dehaene et al., 1998; Draine \& Greenwald, 1998; Greenwald, Draine, \& Abrams, 1996). The robustness of these effects contrasts markedly with findings from procedures that have used nonpracticed subliminal primes (i.e., words that never appeared in visible form in the experiment). Semantic priming effects pro-

This research was supported by National Science Foundation Grant SBR-9710172 and National Institute of Mental Health Grants MH41328, MH01533, and MH57672 to A.G. Correspondence concerning this article should be addressed to R. L. Abrams, Psychology Department, University of Washington, Box 351525, Seattle, WA 98195-1525(e-mail: rlabrams@u.washington.edu). duced by nonpracticed masked words are widely acknowledged to be small in magnitude and difficult to replicate (for reviews, see Draine \& Greenwald, 1998; Forster, 1998).

Second, the just-described pattern of contrasting results from experiments using different procedures has been directly confirmed in recent experiments that compared subliminal priming by practiced and nonpracticed words (Abrams \& Greenwald, 2000; Damian, 2001; Drury \& Klinger, 2000). In these studies, practiced primes produced consistently large subliminal priming effects, whereas nonpracticed primes produced effects that were either nonsignificantly positive or statistically significant but small in magnitude.

Third, practice has been shown not only to enable robust subliminal priming as just described, but also to override effects expected on the basis of existing, well-established semantic knowledge. For example, in a lexical decision experiment in which participants practiced classifying doctor as a visible word, the subliminal word doctor primed both semantically related (nurse) and unrelated (truck) word targets (relative to a baseline condition in which primes were nonwords; Klinger, Burton, \& Pitts, 2000).

Fourth, this practice effect depends on more than just the perceptual encounter with the visible words at time of practice. Practice benefits subliminal priming only when it involves classification into the same categories that will later be used to classify target words in the priming task. When words are repeatedly named but not classi- 
fied in practice, those words remain ineffective as subliminal primes in a semantic classification task (e.g., animate vs. inanimate object; Damian, 2001). ${ }^{1}$

In sum, practice enables effective priming by words that, without practice, apparently receive little analysis. How might practice produce this benefit? Several theories of repetition effects potentially bear on this question (e.g., Morton, 1969; Treisman, 1960). We focus here, however, on a recent suggestion that links subliminal priming by practiced words to the phenomenon of automaticity.

Specifically, it has been suggested that subliminal priming is driven by automation of motor responses (Damian, 2001). According to this view, the critical associations established in practice are between individual words and the responses that are made in categorizing them (word-response mappings). Repeated association of a word with its motor response makes the mapping automatic. When the same word later appears as a subliminal prime, it elicits automatically-outside of attention and awareness - the practiced motoric response.

The chief alternative view is that practice establishes word-to-category mappings rather than word-to-response mappings (cf. Logan, 1990). Word-category mappings are the associations of words to the categories that are used to classify them in the experimental task. These categories may be long-established ones, such as pleasant versus unpleasant meaning, or they may be more ad hoc, such as the word-nonword categories that are used in lexical decision tasks. The word-category interpretation is not necessarily incompatible with automaticity, but it is in contrast with the view that automaticity develops principally for specific motor responses.

The present experiments sought to distinguish between the word-to-response and word-to-category interpretations of the effect of classification practice on subliminal priming. The method was straightforward. We arranged conditions so that the response called for by the practiced word-to-category mapping was opposite to that called for by the practiced word-to-response mapping. Words practiced using one set of response assignments (e.g., left-hand keypress for pleasant words, right-hand keypress for unpleasant words) appeared as subliminal primes in a later task in which classification instructions were reversed from the earlier practice (left hand for unpleasant, right hand for pleasant). If word-to-response mappings underlie subliminal priming, words practiced with one hand should prime targets classified with the same hand (even though those targets belong to the opposite category). Alternatively, the word-to-category view predicts that practiced words should act with their appropriate valence even though the response assignments have been reversed.

\section{EXPERIMENT 1}

Experiments 1 and 2 were designed to test between the predictions of the word-response interpretation (that priming should be determined by practiced response assignment) and the word-category interpretation (that priming should be determined by practiced category). The experiments were carried out in parallel, Experiment 1 at the University of Alabama, and Experiment 2 at the University of Washington. Both used similar designs and procedures, the main difference being that in Experiment 1, masked primes were presented for a slightly longer duration $(50 \mathrm{msec})$ than in Experiment $2(33 \mathrm{msec})$.

\section{Method}

\section{Participants}

Sixty-two University of Alabama undergraduates participated in exchange for credit toward a course requirement. All had normal or corrected-to-normal vision, were fluent in English, and were naive about the hypothesis of the experiment.

\section{Materials}

Two sets of words (A and B) were used as targets and primes, each consisting of 50 words with unambiguously polarized valence: half pleasant in meaning (e.g., happy, warm) and half unpleasant in meaning (scum, kill). All words were presented in uppercase Arial font in black against a white background.

\section{Procedure and Apparatus}

Priming task. The participants first gained practice (four 50trial blocks) at categorizing clearly visible words from one set (A or B) as pleasant or unpleasant in meaning. They responded by pressing either the "a" key with the left hand or the "5" key (on the numeric keypad) with the right hand on a standard computer keyboard. Key assignments in the initial practice phase were counterbalanced, with half the participants receiving $\mathrm{a}=$ unpleasant, $5=$ pleasant, and the remainder receiving the reverse assignment.

The participants then did three 50-trial masked priming blocks in which targets were the same words that had been classified in the initial practice phase (set A or B), and primes were from the same set of words used as targets (however, prime and target were never the same word on any trial). Key assignment was the same as in the initial practice phase.

In the following critical test phase of the experiment, the participants did five 50-trial blocks of a priming task in which targets were words from the set, A or B, that had not been previously presented, and masked primes were from the earlier-practiced set. Key assignment for half the participants was the same as the assignment used earlier (same-keys group); for the other half, it was the reverse of the original assignment (switched-keys group). (Thus in these critical blocks, the words appearing as masked primes had been practiced earlier with either the same or the reverse of the current key assignment.) The first two of these five blocks were identified to the participants as practice, and their data are not included in the analysis.

The sequence of events on priming trials was as follows: fixation point for $300 \mathrm{msec}$, blank text box for $500 \mathrm{msec}$, forward mask for $150 \mathrm{msec}$, prime for $50 \mathrm{msec}$, backward mask for $17 \mathrm{msec}$, then target for $333 \mathrm{msec}$. Forward and backward masks were one of two similar 13-letter strings of consonants (i.e., KQHYTPDQFPBYL). Prime and target were selected at random on each trial to yield a similar number of trials in each of the four possible combinations of prime-target valence. Selection from prime and target sets occurred without replacement, so that each item in the appropriate 50word set appeared exactly once as prime and/or once as target in each 50-trial block.

The target word was followed immediately by an exclamation mark, the 133-msec duration of which defined the response window, an interval during which the participant was instructed to make the keypress response to classify the target as pleasant or un- 
pleasant in meaning. (For a more detailed description of and rationale for the response window procedure, see Draine \& Greenwald, 1998; Greenwald et al., 1996.) To signal to the participants that a response had been made outside the response window, the exclamation mark briefly turned red when the $133-\mathrm{msec}$ window interval ended.

Perceptibility task. After the participants completed the critical priming blocks, they underwent a test of prime perceptibility (three 50-trial blocks) in which they attempted to categorize words masked under the same conditions as in the priming task. Trials in the perceptibility task were identical to priming trials except that the participants were instructed to disregard the exclamation mark and to take as long as necessary to categorize the briefly flashed, masked word. The same key assignment, targets, and primes that had been used in the final, critical priming blocks were used in the perceptibility task. The participants were given three blocks of preliminary practice with enhanced visibility of primes: In the first, the 50-msec primes were displayed in red with no forward or backward masking; in the second, masks were added; and in the third, the display reverted to the normal black. Posttrial error feedback was given in the practice but not the test blocks.

\section{Results}

Data from the two tasks were analyzed by using the regression method (Greenwald, Klinger, \& Schuh, 1995), in which priming-task performance is regressed onto performance in the perceptibility task. Our analysis focuses on the resulting intercept, which, as described in earlier work, provides a statistical test of the hypothesis that occurrence of priming is associated with zero perceptibility of primes in the perceptibility task (Draine \& Greenwald, 1998; Greenwald \& Draine, 1997).

In order to put the data from the two tasks in common terms and to meet the regression method's requirement of a rational zero point for each measure, the data in each task were computed in terms of the signal detection sensitivity measure $d^{\prime}$. Specifically, in both tasks, hits were defined as pleasant-key responses on trials with pleasantvalence primes, and false alarms were defined as pleasantkey responses on trials with unpleasant-valence primes. The following analysis collapsed across two counterbalanced variables: set (whether the words practiced in the initial phase were from Set A or Set B) and order of key assignments (whether the original key assignment was $\mathrm{a}=$ unpleasant, $5=$ pleasant, or vice versa). Neither of these variables had any noticeable effects on priming magnitude, perceptibility $d^{\prime}$, or regression intercept.

Regression analysis of data from the first priming phase-which preceded the response-mapping manipulation, so that both the same-keys and switched-keys groups used the same key assignment as in practice-showed, as expected, a significant intercept for both groups [for the same-keys group, intercept $d^{\prime}=.75, t(29)=9.05, p<$ .001 ; for the switch group, intercept $d^{\prime}=.65, t(29)=$ $8.15, p<.001]$. The difference between the size of these intercept effects was not statistically significant, as would be expected $[t(58)=0.87, p=.19]$. These data replicate the standard finding of robust subliminal priming from words practiced earlier as visible targets.

Theory-relevant findings come from the second priming phase. Figure 1 shows priming data from this phase (the critical blocks) regressed onto data from the perceptibility task for the same-keys group (Figure 1A) and the switched-keys group (Figure 1B). As can be seen, both groups showed significant subliminal priming [for the same-keys group, intercept $d^{\prime}=.39, t(29)=7.25, p<$ .001 ; for the switched-keys group, intercept $d^{\prime}=.33$, $t(29)=6.07, p<.001]$. These two intercepts do not differ significantly in magnitude $[t(58)=0.78, p=.22]$. Thus, switching key assignment between practice and test had little or no effect on priming. The practiced sublim-

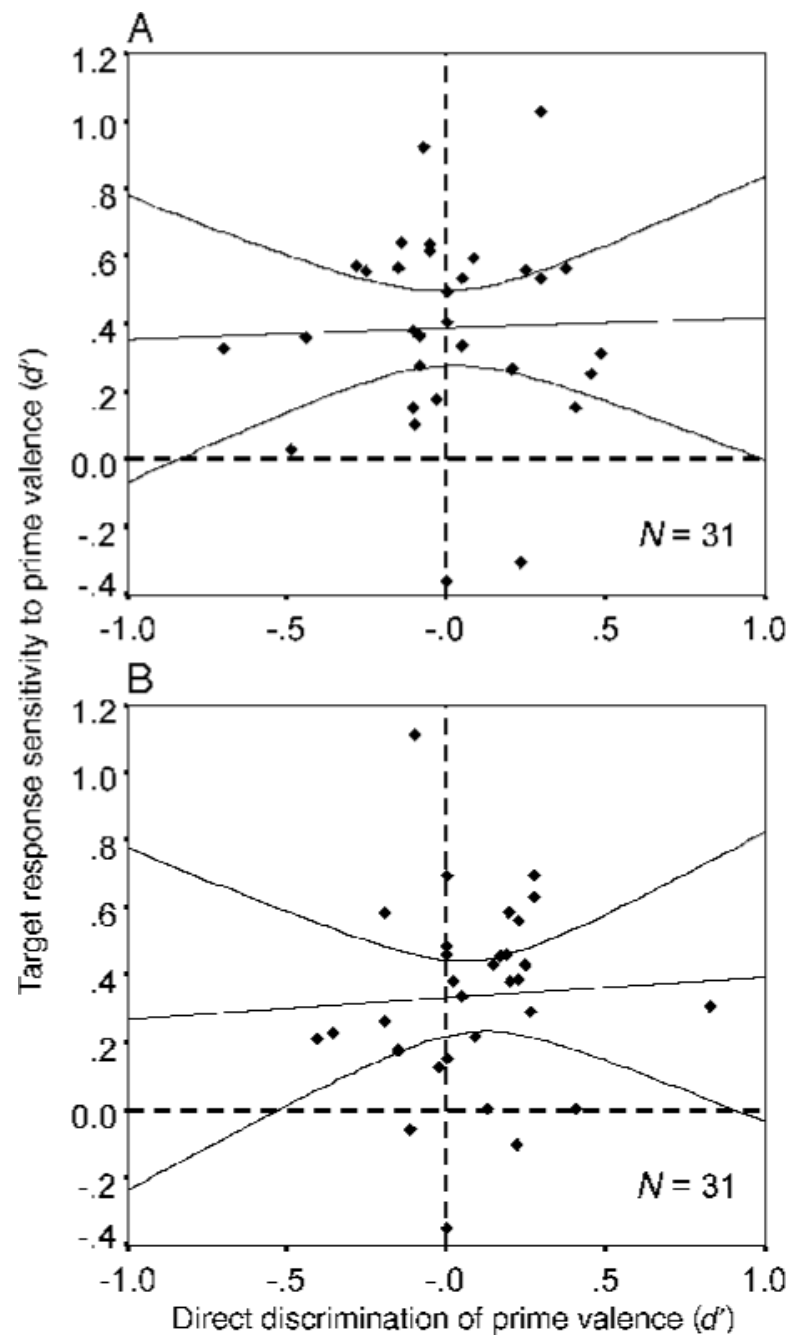

Figure 1. Regression analyses for participants in (A) the samekeys group (response assignments the same in practice and test) and $(B)$ the switched-keys group (response assignments reversed between practice and test) in Experiment 1. Data points represent individual participants. The extent to which masked words functioned with their appropriate valence (unpleasant or pleasant) in the priming task is plotted as a function of performance in classifying them in the perceptibility task. The regression function (with curves indicating a $95 \%$ confidence interval) thus shows the relationship between priming and the ability to consciously perceive primes. Priming is statistically significant and is interpreted as unconscious in operation when the curve for the lower $95 \%$ confidence interval passes above the origin. 
inal words in the switched-keys group acted with their appropriate valence, not with the (now inappropriate) valence that was associated with their earlier-practiced key assignment. (If the latter had occurred, the regression intercept in Figure 1B would have been negative.) This result is consistent with the view that priming was driven by word-to-category mapping, not word-to-response mapping.

\section{EXPERIMENT 2}

As noted earlier, Experiment 2 was conducted in parallel with Experiment 1. Differences in procedures represent only the usual types of variations between different laboratories investigating the same problem. In addition to providing a replication of Experiment 1, Experiment 2 included two features that extended Experiment 1. First, in Experiment 2, the primes were displayed for a shorter duration-33 rather than $50 \mathrm{msec}$. Second, the $\mathrm{A}$ and $\mathrm{B}$ word sets in Experiment 2 had no letters in common, eliminating the possibility that practice on the second set of targets might interfere at the subword level with processing of masked primes. (Earlier research has shown evidence for such possible interference; Abrams $\&$ Greenwald, 2000.) Other more minor differences between the two experiments are described below.

\section{Method}

\section{Participants}

Fifty University of Washington undergraduates participated in exchange for credit toward a course requirement. All had normal or corrected-to-normal vision, were fluent in English, and were naive about the hypothesis of the experiment.

\section{Materials}

The two sets of primes and targets each consisted of 12 words, 6 pleasant and 6 unpleasant in meaning. All words were four letters long. The chief difference from Experiment 1 was that the stimuli in Experiment 2 were constructed so that words in Set A had no overlap of letters with words in Set B. (Examples from Set A are the unpleasant words barf and damp and the pleasant words food and warm; from Set B, geek, ugly, and luck, nice.) Because subliminal analysis may operate largely at the subword level, words classified as targets may influence the analysis of subsequently appearing masked primes with which they share letters (e.g., classifying best earlier as a positive target could interfere with the negative valence of the subliminal prime mess). By having targets and primes share no letters, we should have eliminated this potential interference.

Masks in Experiment 2 were composed not of whole letters, but of letter fragments (again, in order to avoid any influence on prime processing at the letter level). The two masks (forward and backward) on each trial were drawn without replacement from a set of eight, each of which had the same letter fragments distributed in different arrangements across a rectangular area slightly larger than the largest prime word.

\section{Procedure and Apparatus}

The procedure and apparatus were similar to those of Experiment 1 . The participants gained initial practice classifying one set of words with one of the two key assignments (four blocks of trials; all blocks in Experiment 2 consisted of 48 trials). They then received two blocks of practice with the other set of words, with ei- ther the same or reversed key assignment. (Unlike in Experiment 1, the primes in all practice blocks were the letter string, XXXX. In Experiment 2, the initial priming phase was omitted in which targets, primes, and key assignment were the same as in initial practice.) The participants then received six critical priming blocks with the targets and key assignment from the two most recent blocks of practice and masked primes from the words classified in the initial practice phase.

In Experiment 2, the sequence of events on priming trials was as follows: fixation point for $500 \mathrm{msec}$, forward mask for $300 \mathrm{msec}$, prime for $33 \mathrm{msec}$, backward mask for $33 \mathrm{msec}$, target for $333 \mathrm{msec}$. The stimuli were selected in the same way as in Experiment 1. The temporal center of the 133-msec response window interval was initially set at $400 \mathrm{msec}$ following target onset, but, unlike in Experiment 1 , the program controlling the experiment advanced or delayed the window center by $33 \mathrm{msec}$ after each block in order to maintain an error rate of approximately $35 \% .^{2}$ The responsewindow exclamation mark turned red briefly when a response was successfully made within the window interval. Feedback on accuracy of responding, response latency, and response-window accuracy was given at the end of each block.

As in Experiment 1, the critical priming blocks were followed by a test of prime perceptibility (four blocks), which used the targets, primes, and key assignment from the critical priming blocks. Trials were identical to priming trials except that the participants could disregard the earlier instruction to respond while the exclamation mark was present. Practice for the perceptibility task consisted of (1) a block in which the masked words were displayed for $83 \mathrm{msec}$ (a duration that earlier testing had established as adequate for correct classification on nearly all trials) and (2) a block in which duration of the masked words was reduced to $67 \mathrm{msec}$. Posttrial error feedback was provided in the practice, but the not the test, blocks.

\section{Results}

Figure 2 shows that, as in Experiment 1, practiced words acted as effective subliminal primes even when response mapping was reversed between practice and priming. In Figure 2, priming in the critical priming blocks is shown regressed onto perceptibility of primes in the perceptibility task. (As in Experiment 1, the effect of order of sets and order of key assignments was nonsignificant, and those counterbalanced manipulations were ignored in the present analysis.) The regression intercept was significantly positive for both groups [same-keys group, intercept $d^{\prime}=.16, t(21)=5.57, p<.001$; switched-keys group, intercept $\left.d^{\prime}=.12, t(25)=3.81, p<.001\right]$. Further, these intercepts did not differ significantly in magnitude $[t(48)=1.13, p=.13]$. These results corroborate those of Experiment 1, indicating that subliminal priming arises largely through unconscious categorization (word-tocategory mappings), not automation of specific word-toresponse mappings. ${ }^{3}$

The results of Experiment 2 also strengthen a secondary finding of Experiment 1 involving the persistence of the effects of practice in enabling subliminal priming. In both experiments, the critical priming blocks followed initial practice after an interval of practice with different words lasting about 4-5 min. Because the critical priming blocks lasted about 6-8 min, the mean interval between a word's last practice classification and its reappearance as a subliminal prime was about $8-10 \mathrm{~min}$. This is considerably longer than the practice-to-priming in- 


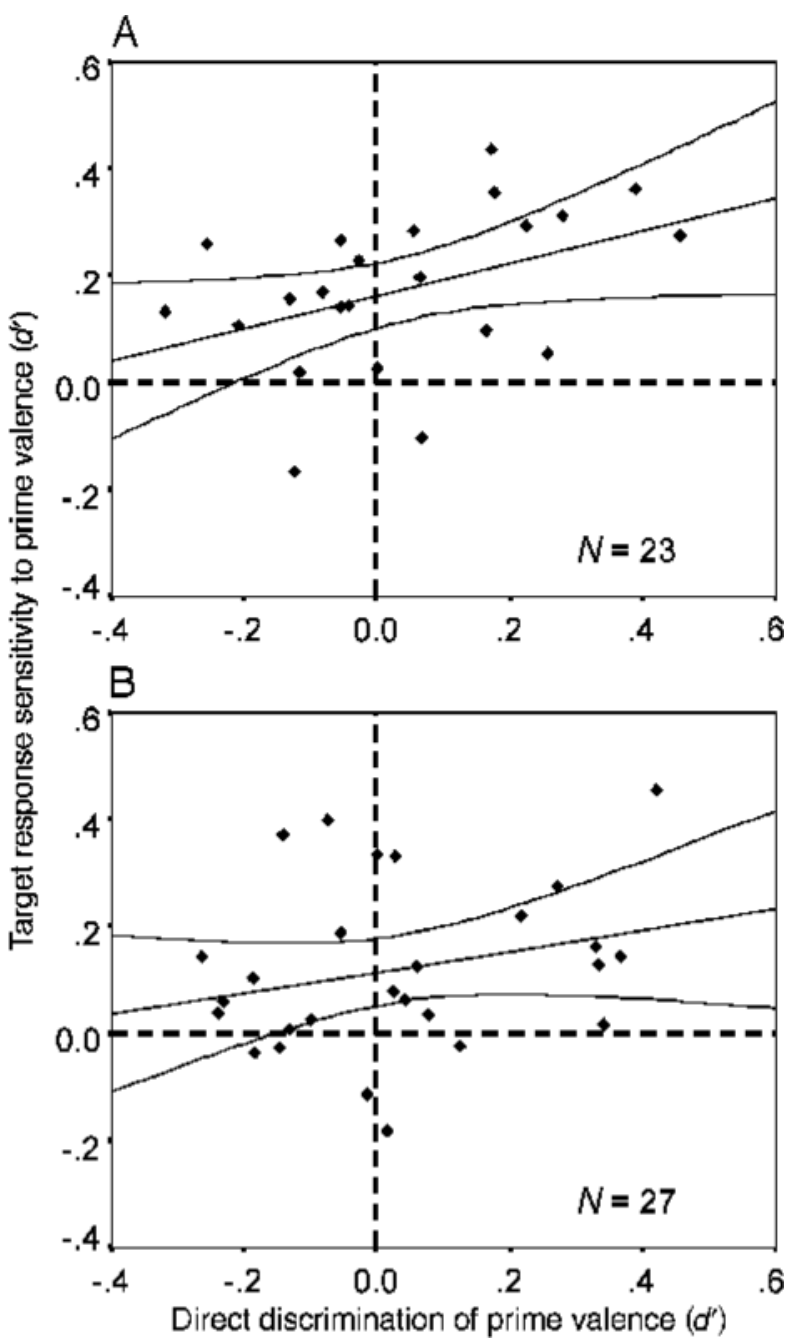

Figure 2. Regression analyses for participants in Experiment 2's (A) same-keys and (B) switched-keys groups. As in Figure 1, data points represent individual participants; see that figure for an explanation of the regression analysis.

terval in previous studies involving practiced primes (e.g., Draine \& Greenwald, 1998; Greenwald et al., 1996; Klinger et al., 2000). In those studies, words classified repeatedly as visible targets reappeared within tens of seconds as subliminal primes (due to the fact that the same set of words was presented concurrently as targets and primes). The present results demonstrate that subliminal priming can be obtained when (1) the set of primes differs from the set concurrently appearing as targets and (2) an interval of at least several minutes elapses between practice and priming. ${ }^{4}$

\section{GENERAL DISCUSSION}

Experiments 1 and 2 followed up on recent findings that robust subliminal priming is obtained only when the words appearing as primes have been practiced (classi- fied) earlier as visible words (Abrams \& Greenwald, 2000; Damian, 2001; Drury \& Klinger, 2000). Here we tested two contrasting hypotheses about the role of practice. According to one hypothesis, practice makes automatic the mappings between words and the specific motor responses used to classify them; the same words appearing later as masked primes elicit those responses automatically (but do not activate semantic category; Damian, 2001). According to the alternative hypothesis, subliminal words activate semantic categories via wordcategory mappings established or strengthened in practice. The results of Experiments 1 and 2 clearly support the latter hypothesis. The subliminal priming that was obtained in both experiments was unaffected by reversal of response assignments between practice and the priming task. If the word-response hypothesis was correct, then, after reversal of assignments, primes should have activated inappropriate motor responses (e.g., words classified as pleasant-meaning in practice with a righthand keypress should have continued to activate a righthand response, even though that response was now associated with unpleasant-meaning words). But primes functioned appropriately, despite reversal of assignments, as representatives of their practiced semantic category. Priming by subliminal words therefore appears to be driven predominantly by activation of practiced word-category mappings.

\section{Validity of the Regression Method}

The conclusion that the priming in the present experiments was subliminal rests in part on analyses using the regression method developed by Greenwald and colleagues (Greenwald \& Draine, 1997; Greenwald et al., 1995). The regression method has been successfully applied in a number of studies of subliminal priming (e.g., Abrams \& Greenwald, 2000; Draine \& Greenwald, 1998; Greenwald et al., 1996). Its validity has also been debated, in detail, in a series of articles (Dosher, 1998; Merikle \& Reingold, 1998) in response to Draine and Greenwald (1998); we refer the reader to those papers for a full account of the points on which the regression method has been questioned. In their responses to those points, Greenwald and Draine (1998) and Klauer, Greenwald, and Draine (1998) provided answers to the major theoretical objections. We restrict the focus of the present discussion to features of datasets that we acknowledge as potentially problematic for the regression method.

Specifically, measurement error in the predictor variable (the perceptibility task) can lead to intercepts that are spuriously large when both the mean of the predictor is substantially greater than zero and the regression slope is significantly positive. When datasets have these features, alternative analyses may be required (cf. Klauer, Draine, \& Greenwald, 1998; Klauer et al., 1998). The present datasets do not have these features. Across the four conditions in the two experiments, only the data from the same-keys group in Experiment 2 reveal a sig- 
nificantly positive slope [slope $=.31 ; t(21)=2.09, p=$ $.049]$. For this group, the mean of the predictor was small and not significantly greater than zero $\left[d^{\prime}=.04, t(22)=\right.$ $0.92, p=.37]$. Further, in three of the four groups, the perceptibility-task mean was not significantly greater than zero, and in the fourth, the significance test yielded $p=.051 .5$ The present data thus show a pattern of significant effects in the indirect measure of prime processing (the priming task) in the absence of effects in the direct measure (the perceptibility task). Such a pattern is well established as a criterion for unconscious processing. It is a problematic criterion, however, in that, to be met, it requires acceptance of a null hypothesis (the hypothesis of no prime processing in the direct measure). The regression method overcomes this problem by taking as the criterion of unconscious processing a rejection of the null hypothesis (i.e., a significantly positive intercept). The fact that the data conform to both criteria provides strong support for the conclusion that the primes in the present experiments operated nonconsciously.

\section{Word-Category Mapping and Unconscious Analytic Capability}

An important question regarding unconscious or unattended processing is the level of complexity at which stimuli are analyzed (e.g., see Greenwald, 1992; Kihlstrom, 1987; Loftus \& Klinger, 1992). For verbal stimuli, this question has usually taken the form: Does unconscious analysis of words operate effectively at the level of whole-word meaning? Numerous findings of subliminal semantic priming over the last two decades have supported the view that, at least in the case of individual words, unconscious analysis of meaning does occur (for reviews of the evidence generally against multiword analysis, see Draine, 1997; Greenwald, 1992).

Several recent findings, however, implicate less complex analytic mechanisms as the basis for subliminal semantic priming. These findings include those reviewed in the introduction showing that robust priming effects require earlier practice with the words appearing later as masked primes. Without practice, subliminal words yield little or no priming, indicating weak or absent wholeword analysis (Abrams \& Greenwald, 2000; Damian, 2001; Drury \& Klinger, 2000). Furthermore, after practice classifying visible words, robust subliminal priming can be obtained from small parts (two or three letters) of those words. Priming is driven by the valence of earlierpracticed subword parts even when these parts are recombined to form whole words of opposite valence (e.g., after classification of agree and pony as pleasant-meaning, agony functions as a pleasant-meaning subliminal prime; Abrams \& Greenwald, 2000).

These recent findings have raised the possibility that subliminal priming is driven by subword parts (not meaning) that activate motor responses (not semantic categories) with which they have become associated in practice. Such a scenario represents a severely limited form of unconscious analysis in which semantic information apparently plays no role at all. The present experiments, however, restore some degree of complexity to unconscious analysis by showing that whatever information is effective in producing subliminal priming, that information activates semantic categories.

\section{REFERENCES}

Abrams, R. L., \& Greenwald, A. G. (2000). Parts outweigh the whole (word) in unconscious analysis of meaning. Psychological Science, 11, 118-124.

Damian, M. F. (2001). Congruity effects evoked by subliminally presented primes: Automaticity rather than semantic processing. Journal of Experimental Psychology: Human Perception \& Performance, 27, 154-165.

Dehaene, S., Naccache, L., Le Clech, G., Koechlin, E., Mueller, M., Dehaene-Lambertz, G., van de Moortele, P., \& Le Bihan, D. (1998). Imaging unconscious semantic priming. Nature, 395, 597600 .

Dosher, B. (1998). The response-window regression method-some problematic assumptions: Comment on Draine and Greenwald (1998). Journal of Experimental Psychology: General, 127, 311-317.

Draine, S. C. (1997). Analytic limitations of unconscious language processing. Unpublished doctoral dissertation, University of Washington.

Draine, S. C., \& Greenwald, A. (1998). Replicable unconscious semantic priming. Journal of Experimental Psychology: General, 127, 286-303.

Drury, S., \& Klinger, M. R. (May, 2000). Repetition priming in unconscious perception. Paper presented at the annual meeting of the Midwestern Psychological Association, Chicago.

FORSTER, K. I. (1998). The pros and cons of masked priming. Journal of Psycholinguistic Research, 27, 203-233.

Greenwald, A. G. (1992). New Look 3: Unconscious cognition reclaimed. American Psychologist, 47, 776-779.

Greenwald, A. G., \& Draine, S. C. (1997). Do subliminal stimuli enter the mind unnoticed? Tests with a new method. In J. Cohen \& J. Schuler (Eds.), Scientific approaches to consciousness: 25 th Carnegie Symposium on Cognition (pp. 83-108). Hillsdale, NJ: Erlbaum.

Greenwald, A. G., \& Draine, S. C. (1998). Distinguishing unconscious from conscious cognition-reasonable assumptions and replicable findings: Reply to Merikle and Reingold (1998) and Dosher (1998). Journal of Experimental Psychology: General, 127, 320-324.

Greenwald, A. G., Draine, S. C., \& Abrams, R. L. (1996). Three cognitive markers of unconscious semantic activation. Science, 273, 1699-1702.

Greenwald, A. G., Klinger, M. R., \& Schuh, E. S. (1995). Activation by marginally perceptible ("subliminal") stimuli: Dissociation of unconscious from conscious cognition. Journal of Experimental Psychology: General, 124, 22-42.

Kinlst Rom, J. (1987). The cognitive unconscious. Science, 237, 14451452.

Klauer, K., Draine, S. C., \& Greenwald, A. G. (1998). An unbiased errors-in-variables approach to detecting unconscious cognition. British Journal of Mathematical \& Statistical Psychology, 51, 253267.

Klauer, K., Greenwald, A. G., \& Draine, S. C. (1998). Correcting for measurement error in detecting unconscious cognition: Comment on Draine \& Greenwald (1998). Journal of Experimental Psychology: General, 127, 318-319.

Klinger, M. R., Burton, P., \& Pitts, S. (2000). Mechanisms of unconscious priming: I: Response competition, not spreading activation. Journal of Experimental Psychology: Learning, Memory, \& Cognition, 26, 441-455.

Loftus, E. F., \& KLINGER, M. R. (1992). Is the unconscious smart or dumb? American Psychologist, 47, 761-765.

Logan, G. D. (1990). Repetition priming and automaticity: Common underlying mechanisms? Cognitive Psychology, 22, 1-35. 
Merikle, P. M., \& Reingold, E. M. (1998). On demonstrating unconscious perception: Comment on Draine and Greenwald (1998). Journal of Experimental Psychology: General, 127, 304-310.

MorTON, J. (1969). The interaction of information in word recognition. Psychological Review, 76, 165-178.

Treisman, A. (1960). Contextual cues in selective listening. Quarterley Journal of Experimental Psychology, 12, 242-248.

\section{NOTES}

1. Several studies including Damian (2001) have reported robust subliminal priming from words presented in one visual form (e.g., uppercase) after having been practiced in another (lowercase; Draine \& Greenwald, 1998; Greenwald, Draine, \& Abrams, 1996). Because upper- and lowercase letters share only some visual features, this is further evidence that practice does not simply facilitate processing at the level of raw perceptual information.

2. The interval between target onset and window center was increased by $33 \mathrm{msec}$ if in the just-finished block (1) the error rate in classifying targets was greater than or equal to $45 \%$ or (2) the error rate was greater than or equal to $35 \%$ and mean response latency was greater than the center value of the window in that block plus $100 \mathrm{msec}$. The interval was decreased by $33 \mathrm{msec}$ if error rate was less than or equal to $20 \%$ and mean latency was less than or equal to the center value of the window in that block plus $100 \mathrm{msec}$.

3. The regression intercepts in Experiment 2 were considerably smaller than in Experiment 1. A likely explanation for this difference is the shorter duration of the primes in Experiment $2(33 \mathrm{msec}$ vs. $50 \mathrm{msec}$ in Experiment 1). A similar difference in magnitude of subliminal priming as a function of prime duration was reported by Greenwald, Draine, and Abrams (1996).

4. The results of Experiment 1 suggest that although the effects of practice are sufficiently durable to produce priming after a severalminute interval, they also undergo some decay. The regression intercept for the first priming phase in Experiment 1 (in which primes were being concurrently practiced as targets) was approximately twice as large as that for the critical priming phase (in which an interval of about 6-8 min had elapsed since they were last practiced as visible words).

5. Experiment 1's same-keys group: $d^{\prime}=.003, t(30)=0.06, p=.95$; switched-keys group, $d^{\prime}=.09, t(30)=2.03, p=.051$. Experiment 2 's same-keys group: $d^{\prime}=.04, t(22)=0.92, p=.37$; switched-keys group: $d^{\prime}=.03, t(26)=0.84, p=.41$.

(Manuscript received September 20, 2000; revision accepted for publication March 27, 2001.) 